\title{
ULTRA HIGH-GRADIENT ENERGY LOSS BY A PULSED ELECTRON BEAM IN A PLASMA
}

\author{
N. Barov, K. Bishofberger, J.B. Rosenzweig, \\ UCLA Dept. of Physics and Astronomy, Los Angeles, CA 90095 \\ J.P. Carneiro* ${ }^{*}$ P. Colestock ${ }^{\dagger}$, H. Edwards, M.J. Fitch ${ }^{\ddagger}$, W. Hartung ${ }^{\S}$, J. Santucci, \\ FNAL, Batavia, IL 60510, USA
}

\begin{abstract}
The plasma wake-field mechanism can be used to couple energy at a high rate from a bunched electron beam into a plasma wave. We will present results from the Fermilab A0 facility where a beam with an initial energy of $14 \mathrm{MeV}$ passes through the plasma to emerge with a much broader energy spread, spanning from a low of $3 \mathrm{MeV}$ to a high of over $20 \mathrm{MeV}$. Over the $8 \mathrm{~cm}$ lenth of the $10^{14} \mathrm{~cm}^{-3}$ plasma, this implies a $140 \mathrm{MeV} / \mathrm{m}$ deceleration and $72 \mathrm{MeV} / \mathrm{m}$ acceleration gradient.
\end{abstract}

\section{INTRODUCTION}

There has been much recent activity in the field of plasma wake-field acceleration (PWFA) [1] [2] [3]. This acceleration scheme has a further advantage when operated in the extremely nonlinear, or blowout regime [4], where the beam is denser than the plasma. Due to the radial outward expulsion of all plasma electrons from the region of the drive and witness beams, the focusing force is radially linear, and the acceleration gradient is independent of radius.

While the acceleration of electrons is the ultimate goal of the PWFA, we find several compelling reasons to concentrate on the energy loss of the drive beam in such a system. The PWFA in the blowout regime is an extremely nonlinear system, and in order to systematically understand it, we must first investigate the coupling of energy from the drive beam into the plasma. This phenomenon has been analyzed recently, with a focus on the question of the saturation of the plasma response for very high charge [5]. Further, because it depends on fewer experimental variables, the energy loss can be a particularly good diagnostic of the nonlinear beam-plasma interaction. Also, the desire to increase the overall efficiency of the PWFA system requires that we understand in detail what happens when a reasonably large fraction of the drive beam energy is transfered to the plasma. Finally, we note that the coupling of large wave energy densities into plasma is also of interest for other applications, such as production of $\mathrm{x}$-rays from highly ionized plasma species excitation [3].

In the limiting case of infinitely short drive pulses, the rate of energy loss by the beam is [5],

$$
\frac{d E}{d z}=N_{b}^{2} e^{2} k_{p}^{2} \ln \left(1.123 / k_{p} a\right)
$$

\footnotetext{
* presently at DESY

presently at LANL

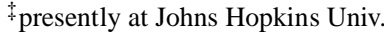

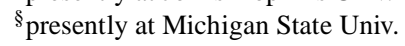

where $N_{b}$ is the number of electrons in the drive beam, $\omega_{p}=c k_{p}$ is the plasma frequency, and $a$ is the beam hard-edge radius which is assumed much smaller than the plasma skin-depth $1 / k_{p}$. Recent work has shown that this result holds true even when the drive beam charge is large enough to cause the plasma electron motion to be relativistic [5].

For finite length bunches, the above expression should be multiplied by a factor of $\exp \left(-\left(k_{p} \sigma_{z}\right)^{2}\right)$, thus implying that the beam energy loss gradient is maximized by choosing the highest density plasma such that $\left.k_{p} \sigma_{z}<1.5\right)$. It should also be noted that for finite bunch length the energy loss (and thus the peak accelerating gradient behind the drive beam) exhibits a saturation behavior, yielding a lower value than predicted by the above equations for a given charge. The relevance of nonlinear saturation effects can be deduced from examining the normalized charge parameter $\tilde{Q}=4 \pi k_{p} r_{e}$. If $\tilde{Q}$ is over 10 , saturation effects become notable, and in fact become dominant for $\tilde{Q}>100$. However, in our case $\tilde{Q} \simeq 1-3$, so we do not expect strong saturation.

\section{EXPERIMENTAL SETUP}

The A0 photoinjector [6] was designed to be the electron source for the TESLA Test Facility accelerator. It features a 1.6 cell copper gun, and a superconducting 9-cell standing wave cavity, both operating at $1300 \mathrm{MHz}$. The machine design specified a low charge regime for FEL experiments, and a high charge $(8 \mathrm{nC})$ regime required for the TESLA parameters.

Using the dipole chicane compressor, the $8 \mathrm{nC}$ beam has been compressed to an RMS bunch length of $0.6 \mathrm{~mm}$ [7], as measured by a Hamamatsu C5680 streak camera looking at OTR light from a thin Al layer mounted on glass.

A hollow cathode plasma source was developed for this experiment. Unlike a traditional hollow cathode arc, where the cathode is heated by the plasma itself, the tantalum cathode is heated by an external DC power supply. This allows for pulsed operation of the working gas (argon) and the arc current (110 A for $0.5 \mathrm{~ms}$ ). The plasma is contained by a solenoidal magnetic field, and occupies an $8 \mathrm{~cm}$ long region. It is operated at a $10^{14} \mathrm{~cm}^{-3}$ nominal density.

The plasma region is bounded on the upstream side by a 10 micron thick aluminum foil, protecting the high vacuum region containing the photoinjector. This foil can also be used as an OTR surface for diagnosing the initial beam size and position.

After the plasma chamber, the beam energy is analyzed 
in an imaging, broadband spectrometer. This system includes a vertically focusing quadrupole, a 145 degree horizontal bend magnet, and a phosphor screen. This spectrometer can only give a time-integrated account of the particle distribution.

\section{RESULTS}

For a compressed case with the charge ranging from 4 to $8 \mathrm{nC}$ per bunch, the mean beam energy with the plasma turned off ranges from 13.6 to $14.8 \mathrm{MeV}$, with the peak intensity at $13.8 \mathrm{MeV}$. With the plasma on, the energy distribution becomes much broader. Although the phosphor screen at the spectrometer output window covers almost a factor of two in energy, the beam energy range is broader than these limits, and it is not possible to capture the entire energy range in a single shot. Figure 1 shows the low end of this range, with a strong signal at $4 \mathrm{MeV}$, and a lower end-point of $3 \mathrm{MeV}$, which is not visible in this frame.

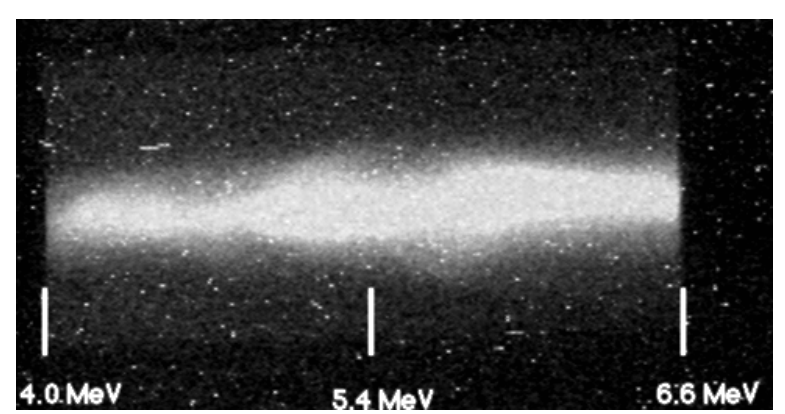

Figure 1. Beam phosphor screen image at spectrometer output window showing decelerated electrons.

We have used the fluid code NOVO [8] to simulate our experimental conditions. This code has been modified to include a super-particle representation of the beam electrons. It is used instead of the analytical expressions given in the Introduction because of the non-Gaussian nature observed in the beam current profile. The streak-camera measured current profile in this case, shows beams with a steep rising edge and a slower falling edge, as expected from the process of chicane compression. This type of beam decelerates at a quicker rate through a plasma than the corresponding Gaussian profile. To approximate the beam in these experiments, we use a split Gaussian model of the current profile, characterized by a $\sigma_{\text {rise }}=0.5 k_{p}$ and $\sigma_{\text {fall }}=2.0 k_{p}$. The simulations also assume a beam charge of $Q=7.0 \mathrm{nC}$, an initial beam spot of $\sigma_{x}=200$ microns, and normalized emittance of $\varepsilon_{n}=45 \mathrm{~mm}$-mrad. The low energy end-point of $E=\gamma m c^{2}=3.2 \mathrm{MeV}$ for this simulation has good agreement with the observed result. However, fluctuations in the bunch length of $+/-25 \%$ can make the result to be as low as $1.2 \mathrm{MeV}$. These fluctuations are caused by the RF system, and cannot be recorded on a shot by shot basis because the streak camera performs a destructive measurement.

The longitudinal phase space from the simulation is shown in Figure 2. In this result, particles in the tail of the beam are accelerated all the way to $31 \mathrm{MeV}$, with an average acceleration gradient of $210 \mathrm{MeV} / \mathrm{m}$. The actual number of particles observed at the highest energies will depend on a number of factors, including the precise shape of the beam tail, transverse effects such as beam head erosion [9], and 3-D effects like hosing [10]. Additionally, some portions of the accelerated tail might be too dilute to definitively stand out above the noise.

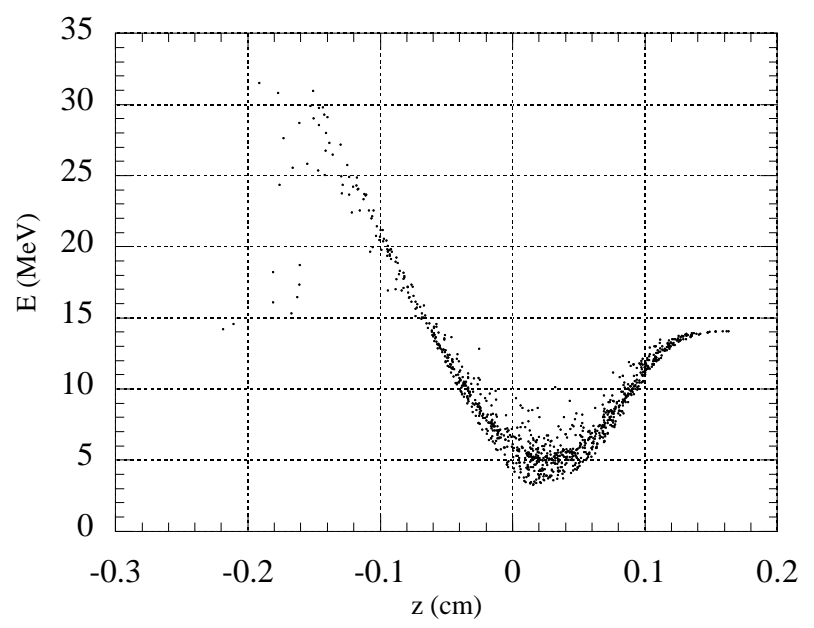

Figure 2. Longitudinal phase space from the simulation.

We unambiguously observe accelerated electrons up to 20.3 MeV, as shown in Figure 3. In order to properly display the high-energy tail on the printed page, the grayscale has been manipulated in a way that makes a large part of the image appear saturated. In order to compute the acceleration gradient, we use a conservative estimate, $14.5 \mathrm{MeV}$, for the starting energy of the electrons. This corresponds to the upper half-maximum of the no plasma energy distribution. The average acceleration gradient is, therefore, at least $72 \mathrm{MeV} / \mathrm{m}$. However, the accelerated tail does not appear to stop at $20.3 \mathrm{MeV}$, which is the maximum range on the spectrometer. In the future, this system will be upgraded to enable the observation of even higher gradients.

Because of the effects of longitudinal space-charge and wake-fields, the bunch length in these experiments was a function of charge. The systematics of the role of charge in bunch length dynamics in both the injector and the compressor are currently under study. The results of this investigation will be used to better model the observed deceleration and acceleration in these experiments.

\section{CONCLUSIONS AND FUTURE WORK}

We have succeeded in coupling a large amount of energy from an electron beam into a plasma wave. After interacting with the plasma, the beam energy distribution is greatly expanded to include a range from $3 \mathrm{MeV}$ to $20.3 \mathrm{MeV}$. To illustrate the intensity of the interaction, it is interesting to quote the amount of power being coupled into the plasma wave during the $266 \mathrm{psec}$ interaction time, which is 115 MW. This power goes mostly into mm-wave energy at the plasma frequency of $90 \mathrm{GHz}$ and its harmonics. As an alternative illustration, we also note that the coherent interac- 


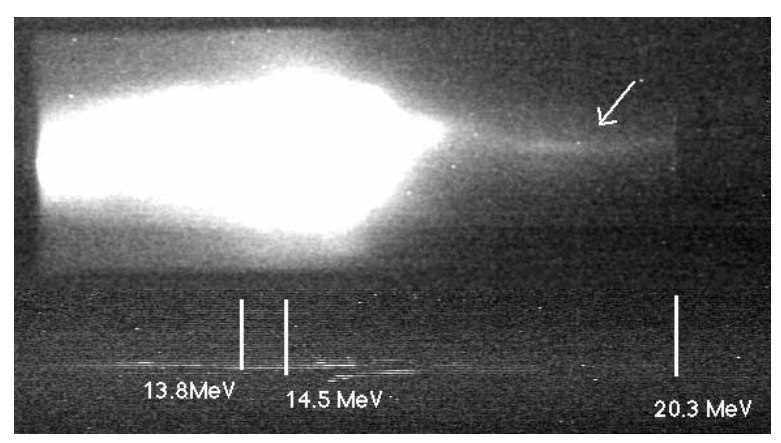

Figure 3. Spectrometer image with plasma on. The accelerated electrons are the whisker-like feature near the arrow.

tion between the beam and the plasma produces a stopping power equivalent to liquid lithium, but at a density 8 orders of magnitude smaller.

The next step for this experiment is to load the plasma wave with a separate witness beam. The technique for doing this involves splitting and optically delaying the photocathode drive laser, which launches the witness beam some tens of picoseconds behind the driver. Although this is easy to achieve for the uncompressed case, the off-crest operation and dipole chicane setup needed for compressed case require a more careful setup. Here, there is the danger of overcompressing, which would tend to put the witness beam on top of the driver. The witness must also have a small enough radius at the start of the plasma as to be captured into the channel. With a suitably intense witness beam, this technique can be used to probe the response of the plasma wake due to beam loading.

A successful witness beam experiment can pave the way for a considerably more ambitious program to demonstrate two-stage acceleration. This would require the creation of two drive beams in adjacent RF buckets, or $0.76 \mathrm{nsec}$ apart. These beams would be separated either with a subharmonic RF cavity, or with a combination of a bend magnet and slightly different energies, and then directed toward two separate plasma interaction zones. Electrons that are accelerated in the first plasma would be transported to the second plasma so that they are collinear and slightly delayed relative to the second drive beam, and thus become further accelerated.

Finally, we note that these experiments clearly demonstrated the worth of compressing a beam, and using a high density plasma (one order of magnitude larger that used in previous uncompressed beam experiments at ANL [1]). The scaling of plasma wave gradient with bunch length $\left(E \sim k_{p}^{2} \sim \sigma_{z}^{-2}\right.$ ) implied from the above discussion has been noted in the literature [1] [11] [12]. It is possible that soon a multi-nC beam may be available at SLAC which is compressed to 12 microns rms. This bunch length implies both the scaling of the wake-fields from the 100's of MV/m level to $10 \mathrm{GV} / \mathrm{m}$ level. It should also be noted that since the normalized charge $\tilde{Q} \simeq 100$ in this case, the relativistic saturation of the plasma response should be observable in such an experiment.

\section{REFERENCES}

[1] N. Barov, et al., "Observation of Plasma Wakefield Acceleration in the Underdense Regime", Phys. Rev. ST-AB 3, 011301 (2000).

[2] P. Muggli, "Status of the Plasma Wakefield Acceleration Experiment at the Stanford Linear Accelerator Center" this proceedings.

[3] S. Russell, et al., "The Los Alamos National Laboratory Plasma Wakefield Accelerator Experiment", this proceedings.

[4] J.B. Rosenzweig, et al., "Acceleration and Focusing of Electrons in Two-dimensional Nonlinear Plasma Wake-fields ", Phys. Rev. E 44 R6189 (1991).

[5] N. Barov, J.B. Rosenzweig, M. Thompson, "Energy Loss of a High Charge Bunched Electron Beam in Plasma", submitted to Phys. Rev. Lett.

[6] J.-P. Carneiro, et al., "First Results of the Fermilab HighBrightness RF Photoinjector", Proceedings of the 1999 Particle Accelerators Conference.

[7] J.-P. Carneiro, "Etude expérimentale du photo-injecteur de Fermilab", PhD thesis, University of Orsay, 2001.

[8] B.N. Breizman, T. Tajima, D.L. Fisher and P.Z. Chebotaev, "Excitation of nonlinear wake field in a plasma for particle acceleration", in Coherent radiation generation and particle acceleration, A. Prokhorov ed., AIP Press, p. 2631992.

[9] N. Barov, M.E. Conde, W. Gai, and J.B. Rosenzweig, "Propagation of Short Electron Pulses in a Plasma Channel", Phys. Rev. Lett. 80, 81, 1998.

[10] J. Krall, and G. Joyce, "Transverse equilibrium and stability of the primary beam in the plasma wake-field accelerator", Phys. Plasmas 2, 1326, 1995.

[11] J.B. Rosenzweig, et al., Nuclear Instruments and Methods A 410532 (1998).

[12] S. Lee, T. Katsouleas, R. Hemker and W. Mori, Phys. Rev. E 617012 (2000). 